\title{
Plant viruses and bacteriophages for drug delivery in medicine and biotechnology
}

\author{
Anna E. Czapar ${ }^{a}$ and Nicole F. Steinmetz ${ }^{* b, c, d, e, f}$ \\ ${ }^{a}$ Department of Pathology, ${ }^{b}$ Department of Biomedical Engineering, ${ }^{c}$ Department of Radiology, ${ }^{d}$ Department of \\ Materials Science and Engineering, ${ }^{e}$ Department of Macromolecular Science and Engineering, ${ }^{f}$ Division of \\ General Medical Sciences-Oncology Case Western Reserve University Schools of Medicine and Engineering, \\ 10900 Euclid Ave., Cleveland, OH 44106, USA \\ *corresponding author: nicole.steinmetz@case.edu
}

\begin{abstract}
There are a wide variety of synthetic and naturally occurring nanomaterials under development for nanoscale cargo-delivery applications. Viruses play a special role in these developments, because they can be regarded as naturally occurring nanomaterials evolved to package and deliver cargos. While any nanomaterial has its advantage and disadvantages, viral nanoparticles (VNPs), in particular the ones derived from plant viruses and bacteriophages, are attractive options for cargo-delivery as they are biocompatible, biodegradable, and non-infectious to mammals. Their protein-based structures are often understood at atomic resolution and are amenable to modification with atomic-level precision through chemical and genetic engineering. Here we present a focused review of the emerging technology development of plant viruses and phages targeting human health and agricultural applications. Key target areas of development are their use in chemotherapy-, photodynamic therapy-, pesticide-delivery, gene therapy, vaccine carriers, and immunotherapy.
\end{abstract}

\section{Introduction}

Advances in nanotechnology have allowed development of nanoscale materials that can be tailored for delivery of medical cargo to molecular zip codes on specific cells or tissues. In addition to the delivery aspect, nanoparticles improve drug stability and 
pharmacokinetics, and the formulation of therapeutics into nanoparticles often leads to reduced toxicity due to favorable biodistribution (avoiding healthy organs and/or enhanced accumulation at the target site). While the development pipeline is moving rapidly with many novel chemistries and (bio)materials emerging, only a few nanoparticles have been approved for clinical use. Translation of novel drug carriers from bench-to-bedside is a long and costly process and challenges in toxicology and large-scale GMP manufacture remain hurdles to success. Addressing the latter point, biologics offer a promising alternative to synthetic materials, because the genetically controlled design and manufacture provides a high degree of quality control and assurance and scale-up can be achieved through fermentation or farming [1]. In this focused review article, we will highlight the development of bacteriophages and plant viruses as an emerging class of biologics for use in drug delivery.

Viruses can be regarded as natural carrier systems; they have evolved to package, protect, and deliver nucleic acid cargo to host cells. Building on these properties, a number of mammalian viral vectors are under development for gene therapy $[2,3]$. Glybera, a viral vector derived from adeno-associated virus (AAV) was the first gene therapy drug approved for clinical use in 2012 in Europe for the treatment of lipoprotein lipase deficiency [4]. Plant viruses and bacteriophages may offer safety advantages as these systems are non-integrating and non-replicating in mammalian systems. Furthermore, because viruses are protein-based, they are biodegradable and thus have a lower risk of tissue persistence compared to some synthetic materials [5,6]. Plant viruses and bacteriophages can be produced and engineered as viral nanoparticles (VNPs) and non-replicative, virus-like particles (VLPs). The VLPs are a subclass of the VNPs and in the remainder of this article we will refer to viral or virus-like systems as VNPs.

Because viruses comes in many shapes, sizes, and surface properties, they offer a toolbox for the nanomedical engineer to pick and chose the most appropriate platform for a given application $[1,7,8]$. For example, higher aspect ratio materials have propensity to marginate and target the diseased vessel wall [9], while flexuous rods allow penetration deep into tumor tissue [10]; and low aspect ratio materials or icosahedrons allow 
effective interactions with the immune system [11]. Many platforms are known to atomic resolution; the well-characterized surface groups on the exterior and interior capsid surfaces allow genetic and chemical tailoring in a predictable way yielding designer nanoparticles [12]. Additionally, the multidendate nature of the capsids offers an array of surface resides making it possible to introduce multiple ligands and cargos, including therapeutic compounds, contrast agents, and targeting ligands into a single formulation $[13,14]$. Functionalization can be achieved through genetic programming, for example amino acids can be introduced to function as conjugation handles (e.g. [15]) or peptides with specificity for cell surface receptors can be added to give the particle directions to target cancer cells (e.g. [16]). Some viruses are also amenable to physical modifications; TMV, for example, can be transformed from its native rod-shape to a spherical form through heating [17].

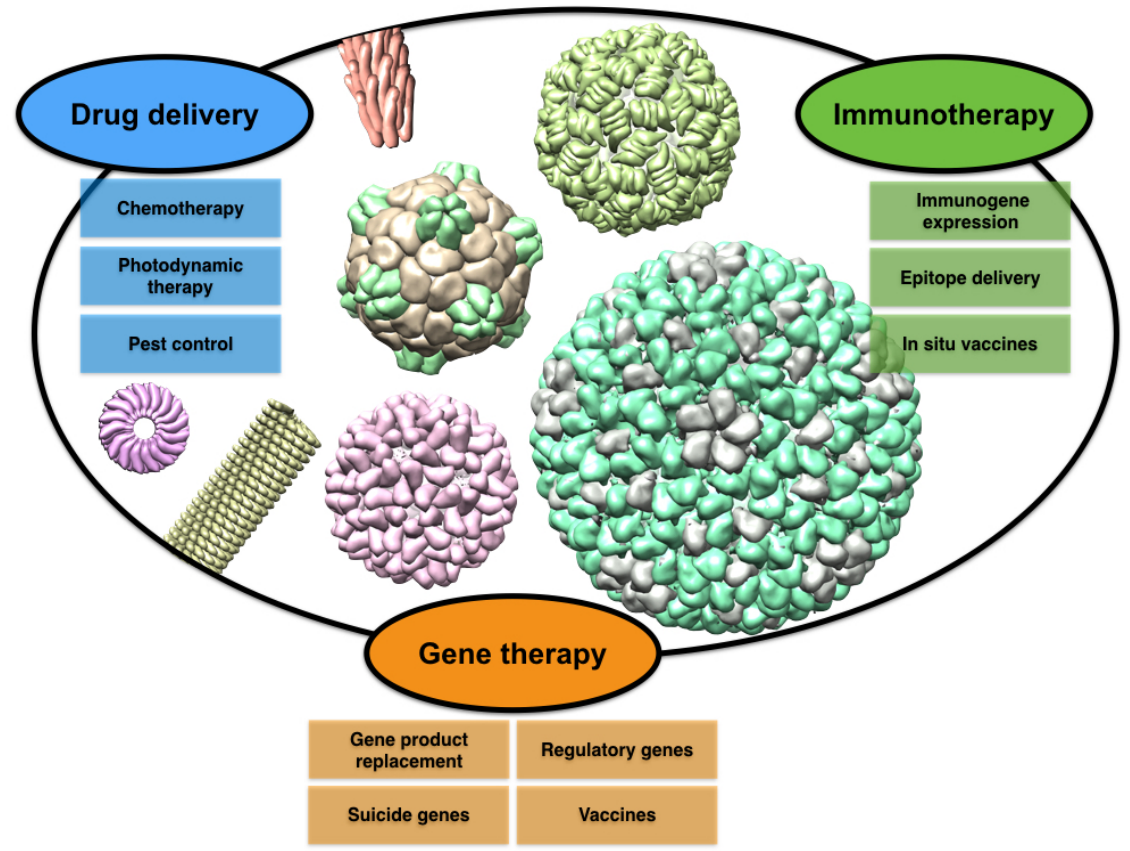

Figure 1. Plant viruses and bacteriophages come in a variety of shapes, sizes, and surface properties and have been explored for a number of biomedical and biotechnology applications. 
For implementation in nanomedicine, the therapeutic cargo is loaded either into the interior cavity of the capsid or conjugated the capsid's interior or exterior surfaces. Targeted delivery of the cargo is then achieved through a combination of (i) choice of carrier shape and (ii) surface chemistry. Carrier size and shape dictate passive targeting to tissues making use of physiopathological features, e.g. enhanced permeability and retention (EPR) effect observed in some tumors [18]. Moreover, active targeting is achieved making use of naturally occurring VNP-cell/tissue interactions (i.e. tropism of cowpea mosaic virus (CPMV) to target and bind to cell-surface vimentin [19]), or active targeting strategies achieved by incorporation of antibodies [20], aptamers [21], or peptides [22] into the capsid scaffold. With increasing understanding of the underlying biology of diseases, there is an ever-expanding capability to target diseased tissue allowing the development of personalized medicine. Figure 1 depicts a few of the plant virus and bacteriophage platforms discussed in this article and provides an overview of the applications that these are developed for.

\section{Drug delivery}

Chemotherapy. VNPs have been explored as nanocarriers for a number of clinically approved and experimental chemotherapeutics. Conjugation or complexation of the therapeutic compound to VNPs can have several advantages including improvement of pharmacokinetics and advantageous biodistribution enabling enhanced drug delivery to the disease site while overcoming off-target effects. Passive and active targeting strategies as mentioned above allow tailored tissue specificity. Several VNP platforms have been developed as a carrier for the anthracycline doxorubicin. In the clinic doxorubicin is used to treat a variety of cancer types including breast, ovarian prostate, head and neck, and thyroid cancer, among others. High off-target toxicity of the drug however, limit the administered dose, and thus reduce treatment efficacy [23]. Liposomal formulation of doxorubicin is one of the few examples of nanoparticle-based drugs used in the clinic today [24]. Examples of VNP-doxorubicin complexes include high aspect ratio TMV-based nanotubes as well as TMV-derived disk-shaped nanoparticles, both of which have been conjugated with doxorubicin through covalent attachment [25-27]. 
Spherical TMV nanoparticles have also been infused with doxorubicin through mixing of doxorubicin and TMV during the heat-mediated rod-to-sphere shape transition. Either method yielded doxorubicin-loaded TMV formulations that exhibited cytotoxicity against cancer cells matched to that of free doxorubicin when tested in vitro [25]. Doxorubicin has also been encapsulated into folate-conjugated hibiscus chlorotic ringspot virus (HCRSV) following disassembly and reassembly of coat proteins. The folate-targeted doxorubicin-loaded HCRSV outperformed free drug in terms of drug uptake and efficacy in cancer cell lines expressing folic acid receptor [28]. Another example is cucumber mosaic virus (CMV); here doxorubicin was infused and trapped inside the capsid through non-covalent loading into the encapsidated RNA genome, leading to increased drug concentration in ovarian cancer tissue and decreased cardiotoxicity in a Balb/C nude mouse model [29]. Similarly, the reversible pore-opening process of the red clover necrotic mosaic virus (RCNMV) capsid allowed loading and release of doxorubicin (as well as other drugs) in a pH- and divalent cation-dependent manner [30]. More recently, we also demonstrated the non-covalent complexation of doxorubicin to high aspect ratio, flexuous potato virus X (PVX); the formulation exhibited enhanced efficacy vs. free drug in a mouse model of triple negative breast cancer [Le, Lee, Steinmetz et al, in review].

While doxorubicin is a good entry point to the field of drug delivery, allowing comparison of the different platform technologies, newer approaches and alternate nanoparticle-drug formulations delivering more potent drugs are urgently needed to overcome drug resistance. Toward this goal, we recently demonstrated TMV-assisted delivery of the drug candidate phenanthriplatin (Figure 2). Phenanthriplatin is a cisplatin derivative up to $40 \mathrm{x}$ more potent than contemporary platinum therapeutics. It has also been shown to be highly effective, even among platinum resistant cell lines [31]. To enable its delivery to tumors, we established a non-covalent drug loading protocol yielding therapeutic TMV carrying 2,000 phenanthriplatin moieties in its central channel. Loading was achieved making use of electrostatic-driven assembly (the dication of phenanthriplatin interacts with the negatively-charged interior of TMV). Phenanthriplatin-loaded TMV outperformed both free phenanthriplatin and the current 
standard of care, cisplatin, when used to treat a mouse model of triple negative breast cancer [32].

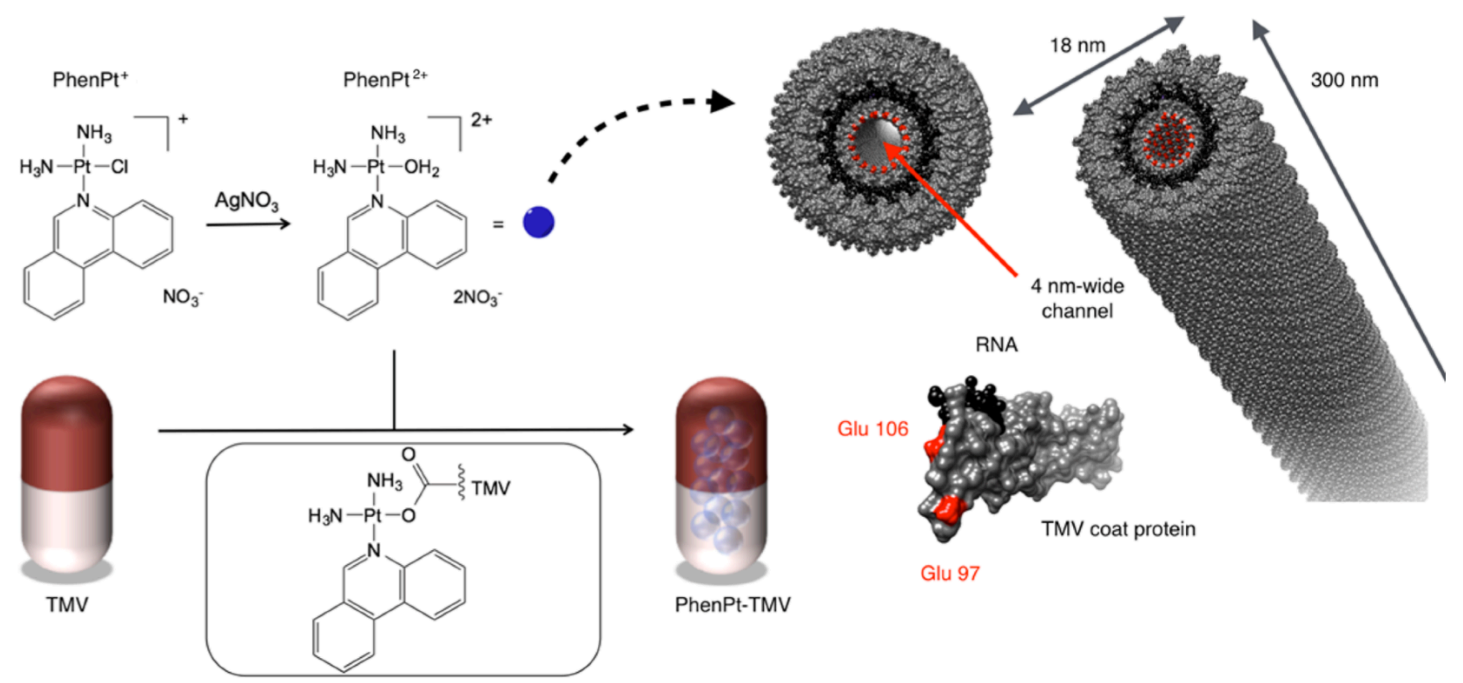

Figure 2. Schematic for loading of phenanthriplatin into TMV. Reprinted with permission from Czapar et al.: Tobacco Mosaic Virus Delivery of Phenanthriplatin for Cancer therapy in ACS Nano [32]. Copyright (2016) American Chemical Society.

Photodynamic therapy. Another avenue is the use of VNPs for the delivery of photodynamic therapies (PDT), an emerging therapeutic regimen used to treat infectious disease and cancer. PDT relies on photosensitizers, often derived from porphyrins, which produce reactive oxygen species (ROS) following activation by specific wavelengths of light. A targeted delivery of PDT allows for increased clinical applications through reduction of host-cell/non-target tissue damage. Several VNP platforms are undergoing development of PDT. Staphylococcus aureus, for example, was treated using cowpea chlorotic mosaic virus (CCMV) dual-functionalized with a photosensitizer and a targeting ligand. Selective delivery of PDT to this infectious agent, may provide an alternative treatment to treat persistent biofilm infections [33]. PDT has also been loaded into the bacteriophages $\mathrm{Q} \beta$ and MS2 while the exterior surface was decorated with targeting ligands for targeted cancer therapy. In brief, aptamer-targeted MS2-delivered PDT allowed for preferential killing of the Jurkat leukemia cell line [21]. Similarly, glycanconjugated Q $\beta$ delivering PDT agents enabled highly specific CD22+ B cell lineage targeting and killing. Potential applications are lymphoma cancer therapy as well as the targeting of certain immune disorders [34]. 
Two recent examples used TMV and CPMV to target melanoma. TMV was loaded with cationic photosensitizers using the electrostatically-driven loading protocol described above (see Figure 2) [32]; the resultant PDT-carrier system showed increased cell uptake and cell killing efficiency when tested with melanoma cells in vitro [35]. CPMV modified with a corona of negatively charged dendrons was used to complex and load positively charged PDT agents. This VNP-synthetic hybrid material was effective in killing both a melanoma cell line as well as macrophages - the combined efficacy on cancer cells and tumor-associated macrophages could be a powerful strategy for cancer therapy [36].

Pest control. VNPs can also be used to improve pesticide delivery for agriculture. Currently, tobacco mottle green mosaic virus (TMGMV) is approved by the EPA as a biopesticide for control of the weed tropical soda apple, demonstrating the potential utility of plant virus-based pest control [37]. One pesticide that could be improved through delivery with VNPs is abamectin, a compound with strong activity against a variety of soil dwelling parasitic nematodes. Nematodes cause an estimated $\$ 157$ billion in crop damage annually and, while abamectin is effective in killing these pests, it has extremely poor mobility in the soil, leading to a limited zone of protection. Abamectin was loaded into RCNMV making use of the $\mathrm{pH}$ - and salt-dependent gating mechanism (Figure 3); the RCNMV carrier was shown to be more effective than free abamectin in nematode killing protecting the roots of plants from disease [38]. 


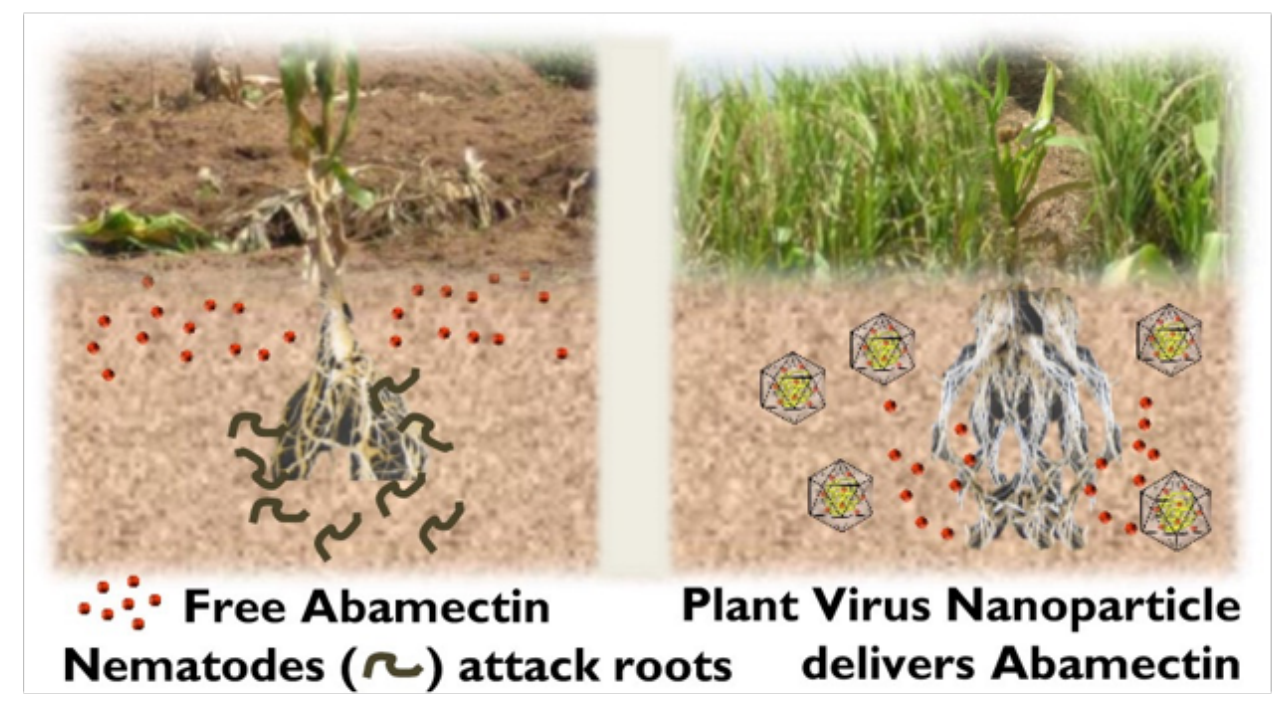

a) Native RCNMV

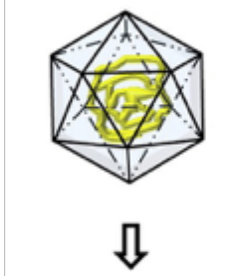

b) Treated with EDTA $\mathrm{pH} 7.5$ to open pores

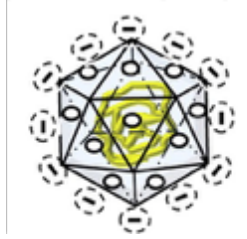

c) Infuse small molecules

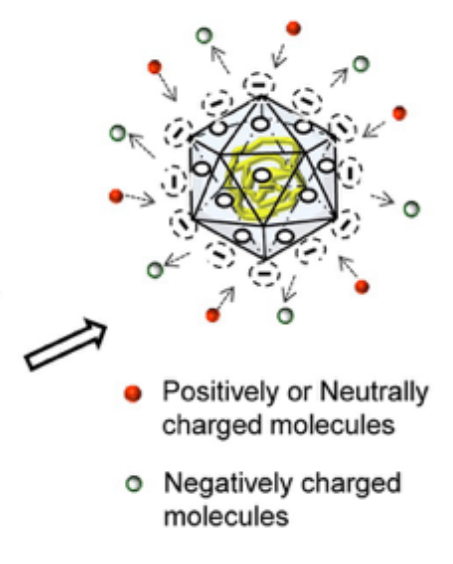

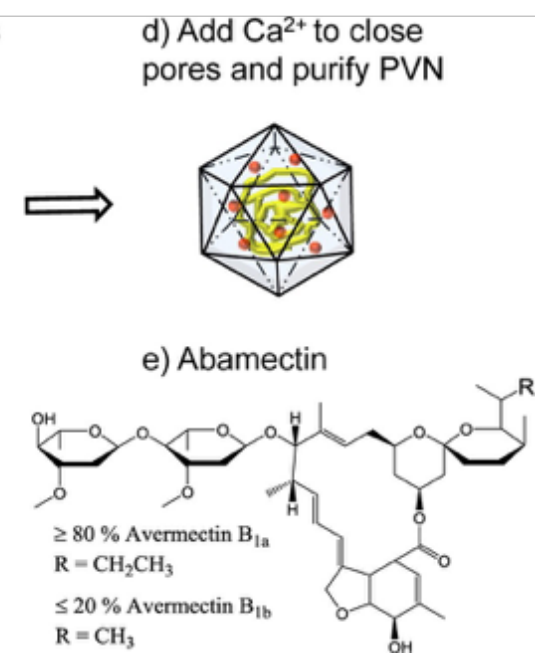

d) Add $\mathrm{Ca}^{2+}$ to close pores and purify PVN

Figure 3. Loading of RCNMV with abamectin for nematode control. Reprinted with permission from Cao J, et al.: Development of Abamectin Loaded Plant Virus Nanoparticles for Efficacious Plant Parasitic Nematode Control in ACS Appl. Mater. Interfaces [38]. Copyright (2016) American Chemical Society.

\section{Gene therapy}

While mammalian viral vectors are already established for gene delivery, in particular in the setting of gene product replacement therapy, newer approaches include the development of plant viruses and bacteriophages targeting diverse applications. A prominent example is the development of CCMV to deliver mRNA cargos. CCMV, like other VNP platform technologies, has the capability to package non-native RNA of a range of lengths and sequences. In a proof-of-concept study, VLPs consisting of a CCMV capsid containing heterologous RNA were delivered to cells using lipofectamine; upon 
cell entry packaged nucleic acids were released in the cytoplasm of mammalian cells leading to expression of the reporter enhanced yellow fluorescent protein (EYFP). Loading of these ssRNAs into the VNP carrier protects the genetic material from degradation during storage and delivery [39]. Bacteriophage M13 has also been used as a nanocarrier for genetic material; through genetic engineering the phage was modified to carry a transgene cassette for herpes simplex virus thymidine kinase (HSVtk), a conditionally lethal enzyme that causes cell death when expressed in the presence of the pro-drug ganciclovir. In addition to the inclusion of this gene, M13 was also modified to express a RGD4C peptide on its nanoparticle surface to enable cancer cell targeting. By combining this targeted phage with a cationic polymer, cell delivery and resulting efficacy was dramatically improved in vitro, demonstrating a potential for M13 as a component of a hybrid synthetic-bionanomaterial [16]. Another area of development is the delivery of mRNAs for vaccination strategies, a topic that we will discuss in the next section (Immunotherapies and vaccines).

Phages have also been developed for gene-silencing applications. A prominent example is the MS2 phage, which has been developed as a carrier for micro-RNAs (miRNAs). Like the plant virus CCMV, the phage has not developed a natural mechanism for trafficking in mammalian cells; however targeting of the nucleic acid cargo to the cytoplasm was achieved through conjugation of the cell penetrating peptide Tat (trans-activator of transcription) from human immunodeficiency virus 1 (HIV-1). In vivo administration of this nanocarrier in a murine model of systemic lupus erthythematous (SLE) achieved a significant increase in the expression of miR146a, a miRNA involved in regulation of the innate immune response and found to be a regulator of autoimmune disorders, myeloproliferation, and cancer. Delivery of the MS2 encapsulated regulatory miR146a was found to have a therapeutic effect as it reduced the total antibody production in the murine SLE model [40].

\section{Immunotherapy and vaccines}

Vaccination by means of gene expression. Plant virus- and bacteriophage- derived VNPs are well suited to use as vaccines and immunotherapy because they are capable of 
stimulating the immune system without the risk of integration or replication - the latter remain safety concerns for attenuated or inactivated vaccines. The size and multivalent nature of VNPs allows for increased immune stimulation, including enhanced B cell activation and uptake by antigen presenting cells. Additionally, even viruses which do not infect mammalian cells stimulate pathogen associated molecular patterns (PAMPs) receptors thus priming the innate immune system, resulting in chemo/cytokine profiles that also stimulate $\mathrm{T}$ cell activation [8]. Building on their ability to interact efficiently with immune cells and their natural capacity to encapsulate nucleic acid cargo, VNPs are undergoing development for delivery and expression of immunogenic transgenes in host cells as a vaccination strategy. In particular, RNA viral vectors have the advantage of inducing high levels of epitope expression in target cells without integration into host DNA. By inducing expression of an antigen, a high level of long-lasting cellular immunity can be achieved. Nevertheless, based on the inherent instability of RNA a delivery system is required. Besides non-viral systems, plant-based VLPs provide an intriguing system to delivery immunogen-encoding RNAs. In one example, a RNA vaccine system was created using a combination of modified RNA-1 from insect virus Flockhouse virus (FHV) and coat proteins from plant virus TMV. In brief, a TMV origin of assembly (TMV OAS) was inserted into the FHV-derived RNA containing coding sequences of the gene of interest. These RNAs were then encapsidated into TMV coat proteins; the assembly is triggered by the presence of the OAS and hybrid VNPs were produced in planta. In a proof-of-concept study using the model immunogen enhanced green fluorescent protein (eGFP) it was demonstrated that such VNP-delivered vaccine strategy yielded an effective humoral response against the target immunogen when tested in mice [41].

Vaccination by means of epitope delivery. In addition to their capability to deliver the coding sequences of immunogens, antigens can also be coded into the capsid proteins, either through genetic fusion or chemical bioconjugation. The multivalent display of the epitope array in combination with the immunogenic nature of the carrier helps to boost epitope-specific B and T cell responses. Developments of VNPs epitope display platform are wide-ranging and applications include vaccination against infectious disease, cancer, 
chronic diseases and addiction. For a comprehensive review on this topic, we would like to refer the reader to the following review [42]. Here we will highlight two recent examples.

In the setting of infectious disease, the bacteriophage P22 has been explored as a platform for a universal influenza vaccine. Influenza nucleoprotein (NP), a protein which is conserved among different strains, was incorporated into the VNP through genetic engineering: a fusion protein was established linking the P22 scaffold protein and NP, coexpression of the fusion protein with the P22 coat proteins in E. coli allowed for production of NP-containing hybrid VNPs. Immunization of mice with purified resultant VNPs elicited a humoral and cellular response with anti-NP antibodies and CD8+ T cells specific to NP produced, resulting in protection of immunized mice against both $\mathrm{H} 1 \mathrm{~N} 1$ and H3N2 intranasal challenges [43]. These data support the utility of the P22-based vaccine as a universal strategy to protect against variable strains of the influenza virus.

The described concepts are applicable to elicit immune response against broad classes of targets. Efforts have also focused on the development of VNPs displaying tumor-associated antigens to elicit therapeutic and prophylactic immunity. One example is the display of an epitope for the idiotypic (Id) immunoglobulin from B-cell lymphoma using the PVX platform technology. Conjugation of the Id epitope to PVX through biotin-streptavidin interactions allowed for a multivalent presentation of the epitope. The multivalent display alongside with immune-stimulation through toll-like receptor-7 (TLR-7) activation based on the encapsidated ssRNA genome of PVX led to enhanced immune stimulation, increased production of anti-Id antibodies, and improved resistance to Id-expressing BCL1 lymphoma challenge when compared to Id-streptavidin alone [44]. The more rigid elongated virus, TMV has also been explored as a vaccine carrier for tumor-specific antigens (e.g. model antigen $\beta$-galactosidase). Through fluorescent tracking, TMV was shown to traffic preferentially to dendritic cells within draining lymph nodes. Enhanced dendritic cell uptake led to improved tumor antigen specific CD8+ $\mathrm{T}$ cell activation. Interesting, unlike adenovirus, TMV did not induce selfneutralizing anti-TMV antibodies, an important factor allowing for administrations of 
subsequent boosting injections [45]. The modifiable nature of these plant viruses, make them a highly versatile choice for a variety of cancer specific antigens.

In situ vaccines. In situ vaccination is a technique where an immune-stimulatory agent is introduced directly into an identified tumor or metastatic site to overcome the immunesuppressive tumor microenvironment and mount an anti-tumor response [46]. With this approach the tumor itself is used as the antigen source and what is introduced is an adjuvant. Using in situ vaccination with mycobacteria has been used over 40 years for bladder cancer [47]. Recently FDA-approved, is TVEC [Amgen], an oncolytic viral therapy based on an attenuated herpes simplex virus engineered to express granulocytemacrophage colony-stimulating factor (GM-CSF) $[48,49]$.

We and others have recently demonstrated that plant VNPs also induce an antitumor immune response when applied as in situ vaccine. First, eCPMV, empty VLPs of CPMV, has been found to be effective in stimulating an anti-tumor immune response when administered into the tumor microenvironment - systemic efficacy and immune memory was demonstrated in mouse models of metastatic melanoma, breast cancer, ovarian cancer, and colon cancer. The in situ vaccination using eCPMV stimulates the innate immune system, in particular eCPMV triggers activation and infiltration of neutrophils, resulting in a chemo/cytokine profile that leads to activation of adaptive immunity. Data indicate that eCPMV in situ vaccination of dermal melanoma in mice elicits a durable anti-tumor response: treated animals were protected from tumor rechallenge (Figure 4) [11]. Similarly to our finding, others have shown that filamentous papaya mosaic virus (PapMV) also exhibited efficacy in treating a mouse model of melanoma when administered intratumorally. The efficacy of PapMV was further improved by synergistic co-administration with dendritic cell vaccination and programmed death ligand 1 (PDL-1) blockade [50]. 

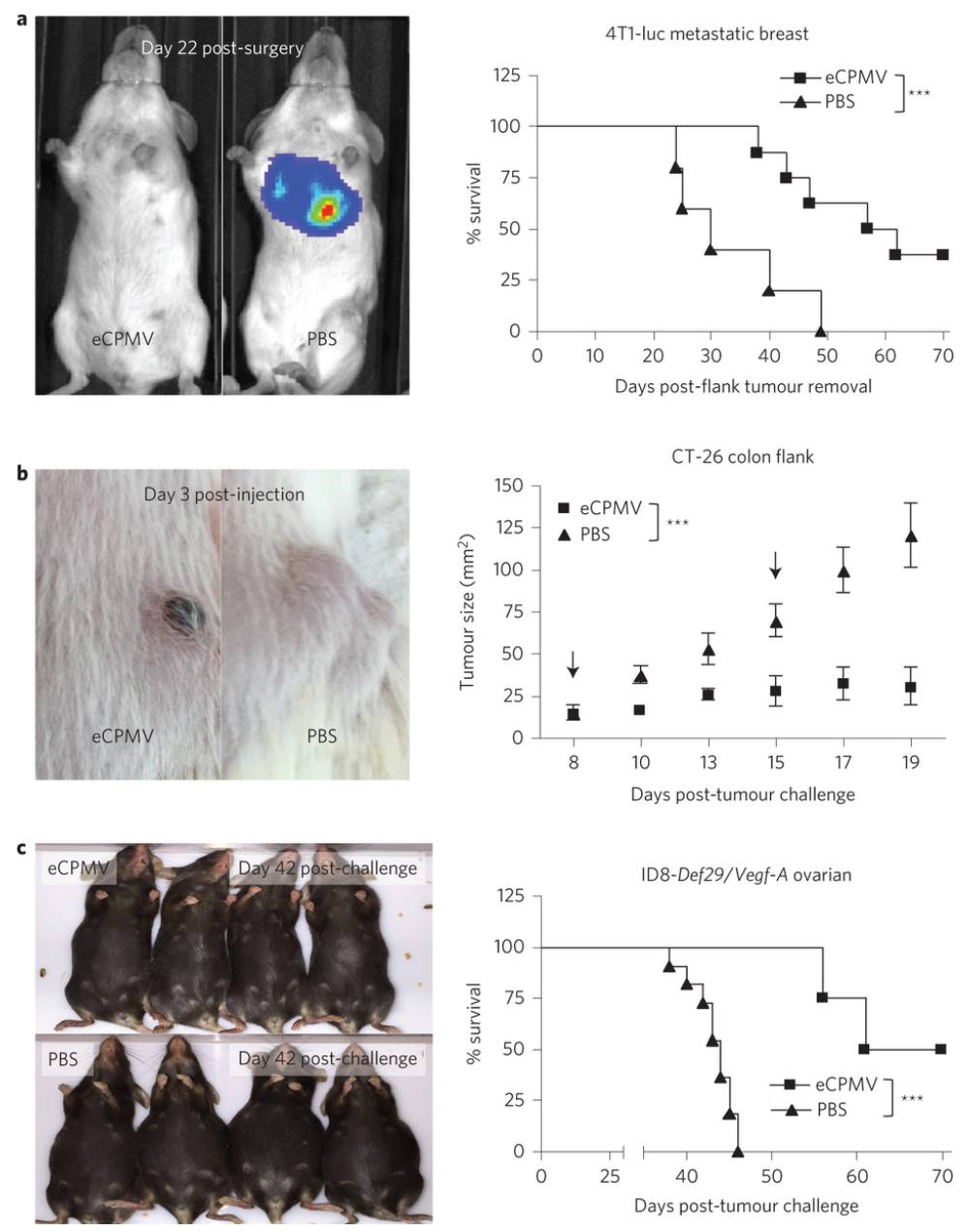

Figure 4. eCPMV administered at the site of the tumor was shown to be effective in treating mouse models of (a) metastatic breast, (b) colon, and (c) ovarian cancer. Reprinted with permission from Lizotte PH, et al.: In situ vaccination with cowpea mosaic virus nanoparticles suppresses metastatic cancer in Nat Nano [11]. Copyright (2015) Nature Publishing Group.

\section{Conclusion}

It is clear that there are many exciting opportunities for potential applications of VNPs in medicine and biotechnology. The field has grown out of its infancy: through collaboration between structural biologists, chemists, physicists, and bioengineers a toolbox of engineering principles has been developed and we are beginning to understand the underlying design concepts to tailor VNPs as cargo-delivery vehicles. Multiple platform technologies are being developed with translational and/or commercial applications on the horizon. Yet there is much work to do to realize the bench-tobedside/field application. First, many works are still being carried out in tissue culture or 
small animal models, which may not mimic human disease in a realistic fashion and therefore do not always provide a realistic and predictive testbed, in particular for toxicology assessment. As for any biologic (as well as synthetic nanomaterials) immunogenicity needs to be carefully evaluated - while a desired property for immunotherapies, immune surveillance must be overcome for therapeutic delivery in which immune-targeting is not desired (e.g. chemotherapy delivery). To some extent, pharmacokinetics and immunogenicity of VNPs can be tailored through conjugation of a polymer stealth coating such as polyethylene glycol or polyoxazoline $[18,52]$ or a camouflage coating such as albumin [53]. Yet newer and more modern approaches are required to make an impact in the clinic. Another consideration is the manufacture of ever more complex formulations harboring cargos, targeting ligands, and stealth/camouflage coatings. Any functionalization applied post-harvest will lower the

yield, increase manufacturing costs, and add the risk of batch-to-batch variability [51]. As the field matures and translation/commercialization become targets in reach, careful design considerations must be taking to yield products that are effective and safe and that have a realistic and cost-effective path of nanomanufacture.

\section{Acknowledgements}

This work was supported in part by a grant from the National Science Foundation (CHE 1306447 to N.F.S.) and by a Research Scholar Award from the American Cancer Society (128319-RSG-15-144-01-CDD to N.F.S.). A.E.C. was supported in part by NIH grants T32 GM007250 and TL1 TR000441. 


\section{References}

1. Yildiz I, Shukla S, Steinmetz NF: Applications of viral nanoparticles in medicine. Current Opinion in Biotechnology 2011, 22:901-908.

2. $\quad * *$ Guenther CM, Kuypers BE, Lam MT, Robinson TM, Zhao J, Suh J: Synthetic Virology: Engineering Viruses for Gene Delivery. WIREs Nanomed Nanobiotechnol 2015, 6: 548 - 558

**Excellent review of methods of engineering of viruses for gene delivery, including genetic engineering and chemical conjugation.

3. Gomez EJ, Gerhardt K, Judd J, Tabor JJ, Suh J: Light-Activated Nuclear Translocation of Adeno-Associated Virus Nanoparticles Using Phytochrome B for Enhanced, Tunable, and Spatially Programmable Gene Delivery. ACS Nano 2016, 10:225-237.

4. Ylä-Herttuala S: Endgame: Glybera Finally Recommended for Approval as the First Gene Therapy Drug in the European Union. Mol Ther 2012, 20:1831-1832.

5. Kaiser CR, Flenniken ML, Gillitzer E, Harmsen AL, Harmsen AG, Jutila MA, Douglas T, Young MJ: Biodistribution studies of protein cage nanoparticles demonstrate broad tissue distribution and rapid clearance in vivo. IJN 2007, 2:715-733.

6. Bruckman MA, Randolph LN, VanMeter A, Hern S, Shoffstall AJ, Taurog RE, Steinmetz NF: Biodistribution, pharmacokinetics, and blood compatibility of native and PEGylated tobacco mosaic virus nano-rods and -spheres in mice. Virology 2014, 449:163-173.

7. Shukla S, Eber FJ, Nagarajan AS, DiFranco NA, Schmidt N, Wen AM, Eiben S, Twyman RM, Wege C, Steinmetz NF: The Impact of Aspect Ratio on the Biodistribution and Tumor Homing of Rigid Soft-Matter Nanorods. Adv. Healthcare Mater. 2015, 4:874-882.

8. $\quad$ Lebel M-È, Chartrand K, Leclerc D, Lamarre A: Plant Viruses as Nanoparticle-Based Vaccines and Adjuvants. Vaccines 2015, 3:620-637.

9. Wen AM, Wang Y, Jiang K, Hsu GC, Gao H, Lee KL, Yang AC, Yu X, Simon DI, Steinmetz NF: Shaping bio-inspired nanotechnologies to target thrombosis for dual optical-magnetic resonance imaging. Journal of Materials Chemistry B: Materials for biology and medicine 2015, 3:60376045 .

10. Shukla S, Ablack AL, Wen AM, Lee KL, Lewis JD, Steinmetz NF: Increased Tumor Homing and Tissue Penetration of the Filamentous Plant Viral Nanoparticle Potato virus X. Mol. Pharm. 2012, 10:33-42. 
11. $\quad * *$ Lizotte PH, Wen AM, Sheen MR, Fields J, Rojanasopondist P, Steinmetz NF, Fiering S: In situ vaccination with cowpea mosaic virus nanoparticles suppresses metastatic cancer. Nat Nano 2015, 11:295-303. **This paper describes the use of eCPMV to stimulate systemic anti-cancer immunity. Through activation of the immune system, survival was dramatically extended in a number of mouse cancer models. Further, the majority of mice re-challenged with tumors on the opposite flank completely rejected the rechallenged tumor.

12. Bruckman MA, Steinmetz NF: Chemical Modification of the Inner and Outer Surfaces of Tobacco Mosaic Virus (TMV). In Characterization of Nanoparticles Intended for Drug Delivery. Edited by McNeil SE. Humana Press; 2013:173-185.

13. Cho K, Wang X, Nie S, Chen Z, Shin DM: Therapeutic Nanoparticles for Drug Delivery in Cancer. Clinical Cancer Research 2008, 14:1310-1316.

14. Bruckman MA, Jiang K, Simpson EJ, Randolph LN, Luyt LG, Yu X, Steinmetz NF: Dual-Modal Magnetic Resonance and Fluorescence Imaging of Atherosclerotic Plaques in Vivo Using VCAM-1 Targeted Tobacco Mosaic Virus. Nano Lett. 2014, 14:1551-1558.

15. Smith ML, Lindbo JA, Dillard-Telm S, Brosio PM, Lasnik AB, McCormick AA, Nguyen LV, Palmer KE: Modified Tobacco mosaic virus particles as scaffolds for display of protein antigens for vaccine applications. Virology 2006, 348:475-488.

16. **Yata T, Lee K-Y, Dharakul T, Songsivilai S, Bismarck A, Mintz PJ, Hajitou A: Hybrid Nanomaterial Complexes for Advanced Phage-guided Gene Delivery. Mol Ther Nucleic Acids 2014, 3:e185-13.

**This paper describes use of M13 bacteriophage that has been genetically altered to express a targeting ligand for gene delivery applications. The incorporation of a genetically engineered targeting ligand and a cationic polymer for increased uptake demonstrates the potential for synthetic and biomaterial hybrids for gene delivery.

17. Bruckman MA, VanMeter A, Steinmetz NF: Nanomanufacturing of Tobacco Mosaic Virus-Based Spherical Biomaterials Using a Continuous Flow Method. ACS Biomater. Sci. Eng. 2015, 1:13-18.

18. Lee KL, Shukla S, Wu M, Ayat NR, Sanadi El CE, Wen AM, Edelbrock JF, Pokorski JK, Commandeur U, Dubyak GR, Steinmetz NF: Stealth filaments: Polymer chain length and conformation affect the in vivo fate of PEGylated potato virus X. Acta Biomaterialia 2015, 19:166-179.

19. Steinmetz NF, Cho C-F, Ablack A, Lewis JD, Manchester M: Cowpea mosaic virus nanoparticles target surface vimentin on cancer cells. 
Nanomedicine 2011, 6:351-364.

20. ElSohly AM, Netirojjanakul C, Aanei IL, Jager A, Bendall SC, Farkas ME, Nolan GP, Francis MB: Synthetically Modified Viral Capsids as Versatile Carriers for Use in Antibody-Based Cell Targeting. Bioconjugate Chem. 2015, 26:1590-1596.

21. Stephanopoulos N, Tong GJ, Hsiao SC, Francis MB: Dual-Surface Modified Virus Capsids for Targeted Delivery of Photodynamic Agents to Cancer Cells. ACS Nano 2010, 4:6014-6020.

22. Pan Y, Zhang Y, Jia T, Zhang K, Li J, Wang L: Development of a microRNA delivery system based on bacteriophage MS2 virus-like particles. FEBS Journal 2012, 279:1198-1208.

23. Tacar O, Sriamornsak P, Dass CR: Doxorubicin: an update on anticancer molecular action, toxicity and novel drug delivery systems. Journal of Pharmacy and Pharmacology 2012, 65:157-170.

24. Marchal S, Hor El A, Millard M, Gillon V, Bezdetnaya L: Anticancer Drug Delivery: An Update on Clinically Applied Nanotherapeutics. Drugs 2015, 75:1601-1611.

25. Bruckman MA, Czapar AE, VanMeter A, Randolph LN, Steinmetz NF: Tobacco mosaic virus-based protein nanoparticles and nanorods for chemotherapy delivery targeting breast cancer. Journal of Controlled Release 2016, 231:103-113.

26. Finbloom JA, Han K, Aanei IL, Hartman EC, Finley DT, Dedeo MT, Fishman M, Downing KH, Francis MB: Stable Disk Assemblies of a Tobacco Mosaic Virus Mutant as Nanoscale Scaffolds for Applications in Drug Delivery. Bioconjugate Chem. 2016, 27: 2480 - 2485

27. Tian Y, Gao S, Wu M, Liu X, Qiao J, Zhou Q, Jiang S, Niu Z: Tobacco Mosaic Virus-Based 1D Nanorod-Drug Carrier via the IntegrinMediated Endocytosis Pathway. ACS Appl. Mater. Interfaces 2016, 8:10800-10807.

28. Ren Y, Wong SM, Lim LY: Folic Acid-Conjugated Protein Cages of a Plant Virus: A Novel Delivery Platform for Doxorubicin. Bioconjugate Chem. 2007, 18:836-843.

29. Zeng Q, Wen H, Wen Q, Chen X, Wang Y, Xuan W, Liang J, Wan S: Cucumber mosaic virus as drug delivery vehicle for doxorubicin. Biomaterials 2013, 34:4632-4642.

30. Cao J, Guenther RH, Sit TL, Opperman CH, Lommel SA, Willoughby JA: Loading and Release Mechanism of Red Clover Necrotic Mosaic Virus 
Derived Plant Viral Nanoparticles for Drug Delivery of Doxorubicin. Small 2014, 10:5126-5136.

31. Park GY, Wilson JJ, Song Y, Lippard SJ: Phenanthriplatin, a monofunctional DNA-binding platinum anticancer drug candidate with unusual potency and cellular activity profile. Proc. Natl. Acad. Sci. U.S.A. 2012, 109:11987-11992.

32. **Czapar AE, Zheng Y-R, Riddell IA, Shukla S, Awuah SG, Lippard SJ, Steinmetz NF: Tobacco Mosaic Virus Delivery of Phenanthriplatin for Cancer therapy. ACS Nano 2016, 10:4119-4126.

**This paper describes the use of TMV increase delivery of a novel cisplatin derivative, phenanthriplatin, to a mouse model of triple negative breast cancer. This is the first paper to describe in vivo delivery of a platinum compound with a plant viral nanoparticle.

33. Suci PA, Varpness Z, Gillitzer E, Douglas T, Young M: Targeting and Photodynamic Killing of a Microbial Pathogen Using Protein Cage Architectures Functionalized with a Photosensitizer. Langmuir 2007, 23:12280-12286.

34. Rhee J-K, Baksh M, Nycholat C, Paulson JC, Kitagishi H, Finn MG: Glycan-Targeted Virus-like Nanoparticles for Photodynamic Therapy. Biomacromolecules 2012, 13:2333-2338.

35. Lee KL, Carpenter BL, Wen AM, Ghiladi RA, Steinmetz NF: High Aspect Ratio Nanotubes Formed by Tobacco Mosaic Virus for Delivery of Photodynamic Agents Targeting Melanoma. ACS Biomater. Sci. Eng. 2016, 2:838-844.

36. Wen AM, Lee KL, Cao P, Pangilinan K, Carpenter BL, Lam P, Veliz FA, Ghiladi RA, Advincula RC, Steinmetz NF: Utilizing Viral Nanoparticle/Dendron Hybrid Conjugates in Photodynamic Therapy for Dual Delivery to Macrophages and Cancer Cells. Bioconjugate Chem. 2016, 27: 1227 - 1235.

37. Charudattan R, Hiebert E: A Plant Virus as a Bioherbicide for Tropical Soda Apple, Solanum Viarum. Outlook Pest Man 2007, 18:167-171.

38. Cao J, Guenther RH, Sit TL, Lommel SA, Opperman CH, Willoughby JA: Development of Abamectin Loaded Plant Virus Nanoparticles for Efficacious Plant Parasitic Nematode Control. ACS Appl. Mater. Interfaces 2015, 7:9546-9553.

39. Azizgolshani O, Garmann RF, Cadena-Nava R, Knobler CM, Gelbart WM: Reconstituted plant viral capsids can release genes to mammalian cells. Virology 2013, 441:12-17. 
40. Pan Y, Jia, Zhang, Zhang, Zhang, Li, Wang L: MS2 VLP-based delivery of microRNA-146a inhibits autoantibody production in lupus-prone mice. IJN 2012, 7: 5957 - 5967.

41. $\quad$ **Zhou Y, Maharaj PD, Mallajosyula JK, McCormick AA, Kearney CM: In planta Production of Flock House Virus Transencapsidated RNA and Its Potential Use as a Vaccine. Mol Biotechnol 2014, 57:325-336.

**This paper describes a highly interesting system of packaging FHV RNA into VLPs derived from TMV coat proteins in plants.

42. Lee KL, Twyman RM, Fiering S, Steinmetz NF: Virus-based nanoparticles as platform technologies for modern vaccines. WIREs Nanomed Nanobiotechnol 2016, 8:554-578.

43. Patterson DP, Rynda-Apple A, Harmsen AL, Harmsen AG, Douglas T: Biomimetic Antigenic Nanoparticles Elicit Controlled Protective Immune Response to Influenza. ACS Nano 2013, 7:3036-3044.

44. Jobsri J, Allen A, Rajagopal D, Shipton M, Kanyuka K, Lomonossoff GP, Ottensmeier C, Diebold SS, Stevenson FK, Savelyeva N: Plant Virus Particles Carrying Tumour Antigen Activate TLR7 and Induce High Levels of Protective Antibody. PLoS ONE 2015, 10:e0118096-16.

45. Kemnade JO, Seethammagari M, Collinson-Pautz M, Kaur H, Spencer DM, McCormick AA: Tobacco mosaic virus efficiently targets DC uptake, activation and antigen-specific $\mathbf{T}$ cell responses in vivo. Vaccine 2014, 32:4228-4233.

46. Hammerich L, Binder A, Brody JD: In situ vaccination: Cancer immunotherapy both personalized and off-the-shelf. Molecular Oncology 2015, 9:1966-1981.

47. Kamat AM, Flaig TW, Grossman HB, Konety B, Lamm D, O'Donnell MA, Uchio E, Efstathiou JA, Taylor JA: Expert consensus document: Consensus statement on best practice management regarding the use of intravesical immunotherapy with BCG for bladder cancer. Nature Publishing Group 2015, 12:225-235.

48. Harrington KJ, Puzanov I, Hecht JR, Hodi FS, Szabo Z, Murugappan S, Kaufman HL, Harrington KJ, Puzanov I, Hecht JR, Hodi S, Szabo Z, Murugappan S, Kaufman HL: Clinical development of talimogene laherparepvec (T-VEC): a modified herpes simplex virus type-1-derived oncolytic immunotherapy. Expert Review of Anticancer Therapy 2015, 15:1389-1403.

49. Andtbacka RHI, Kaufman HL, Collichio F, Amatruda T, Senzer N, Chesney J, Delman KA, Spitler LE, Puzanov I, Agarwala SS, Milhem M, Cranmer L, 
Curti B, Lewis K, Ross M, Guthrie T, Linette GP, Daniels GA, Harrington K, Middleton MR, Miller WH Jr, Zager, JS, Ye Y, Yao B, Li A, Doleman S, VanderWalde A, Gansert J, Coffin RS: Talimogene Laherparepvec Improves Durable Response Rate in Patients With Advanced Melanoma. Journal of Clinical Oncology 2015, 33:2780-2788.

50. Lebel M-È, Chartrand K, Tarrab E, Savard P, Leclerc D, Lamarre A: Potentiating Cancer Immunotherapy Using Papaya Mosaic VirusDerived Nanoparticles. Nano Lett. 2016, 16:1826-1832.

51. McNeil SE: Challenges for Nanoparticle Characterization. In Characterization of Nanoparticles Intended for Drug Delivery. Edited by McNeil SE. Humana Press; 2010:9-15.

52. Bludau H, Czapar AE, Pitek AS, Shukla S, Jordan R, Steinmetz NF: POxylation as an alternative stealth coating for biomedical applications. European Polymer Journal 2016, doi:10.1016/j.eurpolymj.2016.10.041.

53. Pitek AS, Jameson SA, Veliz FA, Shukla S, Steinmetz NF: Serum albumin "camouflage" of plant virus based nanoparticles prevents their antibody recognition and enhances pharmacokinetics. Biomaterials 2016, 89:8997. 